\title{
Atribución de síntomas en pacientes con síntomas médicamente inexplicables. Un estudio de validación y desarrollo de la escala
}

José M. Ramírez-Aranda, ${ }^{1 *}$ Mónica Morales-Ramírez,, Verónica L. Frías-Gómez, ${ }^{2}$ Marco V. Gómez-Meza, ${ }^{3}$ Oralia del Castillo-Guzmán y Cinthia D. López-Mata ${ }^{1}$

${ }^{1}$ Departamento de Medicina Familiar, Facultad de Medicina, Universidad Autónoma de Nuevo León; ${ }^{2}$ Unidad de Medicina Familiar 5, Instituto Mexicano del Seguro Social; ${ }^{3}$ Facultad de Economía, Universidad Autónoma de Nuevo León; ${ }^{4}$ Servicio de Psiquiatría, Hospital Universitario "Dr. José Eleuterio González", Universidad Autónoma de Nuevo León. Monterrey, Nuevo León, México

\section{Resumen}

Introducción: En la atención de pacientes con síntomas físicos médicamente no explicables (SFMNE) es importante lo que el paciente piensa de sus síntomas. Objetivo: Validar propiedades psicométricas de una escala de atribución del síntoma en pacientes con SFMNE y verificar su confiabilidad. Métodos: Se entrevistó a una muestra no probabilística de 400 pacientes adultos, hombres y mujeres, en la consulta de un hospital con medicina familiar, 200 con SFMNE y 200 con patología orgánica concreta. Se diagnosticó a cada grupo con criterios definidos y se aplicó una escala con validez de contenido y de constructo por medio de análisis de componentes principales con rotación varimax. Resultados: La escala quedó integrada por 12 reactivos con dos factores, uno de atribución psicosocial y otro de atribución orgánica del síntoma. El factor de origen psicosocial tuvo una varianza de $49.7 \%$. La prueba de bondad de ajuste mostró que la matriz de correlaciones fue adecuada y la prueba de esfericidad de Bartlett indicó significación estadística $(p<0.0001)$; el alfa de Cronbach fue de 0.841 . Conclusión. La escala mostró una validez de constructo aceptable y buena confiabilidad y estabilidad. Se discuten las implicaciones de estos resultados para la investigación de mediciones futuras.

PALABRAS CLAVE: Síntomas médicamente inexplicables. Estilo de atribución. Psicosocial. Escala.

\section{Symptom attribution in patients with medically unexplained symptoms. A scale development and validation study}

\section{Abstract}

Introduction: In the care of patients with medically unexplained physical symptoms (MUPS) it is important what they think about their symptoms. Objective: To validate the psychometric properties of a symptom attribution scale in patients with MUPS and to verify its reliability. Methods: $A$ non-probabilistic sample of 400 male and female adult patients were interviewed in the outpatient services of a family medicine hospital, 200 with MUPS and 200 with a defined organic pathology. Each group was diagnosed with defined criteria, and a scale with content and construct validity was applied by means of principal component analysis with varimax rotation. Results: The scale was made up of 12 items with two factors, one of symptom psychosocial attribution and others with organic attribution. The psychosocial-origin factor showed a variance of $49.7 \%$. The goodness-of-fit test demostrated that the correlation matrix was adequate, and Bartlett's sphericity test indicated statistical significance $(p<$ 0.0001); Cronbach's alpha was 0.841 . Conclusion. The scale showed acceptable construct validity and good reliability and stability. The implications of these results for future measurement research are discussed.

KEYWORDS: Medically unexplained symptoms. Attribution style. Psychosocial. Scale.

\footnotetext{
Correspondencia:

Fecha de recepción: 01-06-2021

*José M. Ramírez-Aranda

Fecha de aceptación: 24-06-2021

Gac Med Mex. 2022;158:17-23

E-mail: sersabe2010@gmail.com

DOI: 10.24875/GMM.21000336

Disponible en PubMed

0016-3813/@ 2021 Academia Nacional de Medicina de México, A.C. Publicado por Permanyer. Este es un artículo open access bajo la licencia CC BY-NC-ND (http://creativecommons.org/licenses/by-nc-nd/4.0/).
} 


\section{Introducción}

La somatización tiene una larga historia desde la época de Hipócrates; ${ }^{1}$ también se denomina síntomas físicos médicamente no explicados (SFMNE). ${ }^{2}$ Representa un desafío para los médicos debido a su manejo y enfoque. ${ }^{3,4}$

Los pacientes con SFMNE son aquellos cuyos síntomas se caracterizan por quejas somáticas persistentes y en quienes los exámenes y esfuerzos por llegar a un diagnóstico no revelan una patología orgánica. Los síntomas inespecíficos sin explicación médica como motivo de consulta son comunes en atención primaria; su prevalencia oscila entre 40.2 y $49.0 \%{ }^{5}$

No hay consenso sobre cómo diagnosticar a los pacientes con SFMNE. Entre los distintos criterios para hacerlo están los de Reid, que comprenden la evidencia de estudios clínicos negativos, además de la correlación del síntoma inespecífico con un problema psicosocial identificable o el diagnóstico de un síndrome sin etiología orgánica objetiva (fibromialgia, síndrome del intestino irritable o algún otro), ${ }^{6}$ el cual generalmente se observa en la consulta de especialidades como reumatología, medicina interna 0 gastroenterología. ${ }^{6}$

Los pacientes con SFMNE generan altos costos para los sistemas de salud debido al gran uso de recursos y consultas médicas de segundo y tercer nivel. ${ }^{7}$ En atención primaria, el médico de familia realiza un abordaje integral considerando el entorno psicosocial para detectar situaciones estresantes o "crisis" relacionadas con SFMNE. La asociación entre el estrés y la presencia de síntomas inespecíficos ya ha sido establecida. ${ }^{8}$ Aún más, se ha demostrado la efectividad de métodos como la técnica de resolución de problemas. $^{9-11}$ La terapia cognitiva conductual parece ser un enfoque psicosocial práctico; sin embargo, pocos médicos de atención primaria tienen la formación necesaria para ayudar a los pacientes y sus familias. ${ }^{12,13}$

Por otro lado, la causa de los síntomas inespecíficos se ha estudiado durante mucho tiempo. ${ }^{14}$ En 1991, Robbins propuso tres estilos de atribución de los síntomas: física, psicológica y normalizadora o ambiental, aunque los pacientes pueden presentar síntomas en torno a solo dos ejes: el somático y el psicológico. ${ }^{14}$

Medir la atribución de los síntomas no es fácil, además de que hay pocas herramientas para hacerlo, una de ellas es el Symptom Interpretation Questionnaire (SIQ), validado por Aronson en 2006,,$^{15}$ quien trabajó con las tres subescalas del SIQ (psicológica, somática y normalizadora) sugeridas por Robbins. Estos resultados indican que el SIQ tiene un pequeño grado de validez convergente, pero poca validez discriminante.

La literatura muestra que los pacientes con SFMNE tienden a atribuir sus síntomas a disfunciones o enfermedades orgánicas,$^{16}$ debido a que la concepción mecanicista del cuerpo humano se entiende más fácil. En la práctica, un porcentaje considerable de pacientes no reconoce la asociación entre estrés y presencia de síntomas inespecíficos, lo que dificulta cualquier intervención psicoeducativa. Al trabajar exclusivamente con el modelo biomédico, el diagnóstico de SFMNE es un largo proceso de exclusión de una enfermedad orgánica, lo que resulta costoso y frustrante para los médicos y peligroso para los pacientes. ${ }^{12}$

Una intervención del médico puede estar influida por las habilidades de este, pero también por la atribución de los síntomas por parte del paciente, quien dificulta la detección de la depresión y la ansiedad y es más resistente al manejo psicosocial al pensar que el origen del síntoma es orgánico $o$ al tener un estilo de atribución normalizador. ${ }^{17}$

Los pacientes con SFMNE tratados en medicina familiar con una intervención no farmacológica son "pacientes sanos preocupados", quienes, según Smith et al., representan $80 \%$ de los casos, ${ }^{18}$ y que podrían beneficiarse de una intervención psicoeducativa en medicina familiar.

El propósito de esta investigación fue validar un instrumento que mide la percepción del paciente sobre su síntoma y ayuda a facilitar la conexión entre el evento estresante y el síntoma inespecífico, el cual ayudaría al médico de familia a definir y establecer un enfoque más apropiado para el paciente.

\section{Métodos}

Estudio analítico transversal comparativo efectuado en un hospital de tercer nivel con clínica ambulatoria en Monterrey, México. El instrumento para identificar la atribución de síntomas funcionales en pacientes con SFMNE fue desarrollado y validado en español. Un comité de investigación ética aprobó el proyecto en el centro universitario. La escala se diseñó en tres etapas: construcción de los reactivos, pilotaje y validación.

\section{Paso 1, construcción de los reactivos}

Se diseñó una encuesta semiestructurada con base en la respuesta de 50 pacientes, 25 de la clínica 
psiquiátrica y 25 de las clínicas de ortopedia y otras especialidades de "atención de problemas orgánicos". Los pacientes respondieron dos preguntas que guiaron la construcción de la escala: ¿diría usted que sus síntomas son físicos o psicológicos (emocionales)?, seguida de la pregunta abierta ¿por qué piensa esto?

Un panel de expertos de siete especialistas clínicos en psiquiatría, psicología y medicina familiar seleccionaron por consenso, mediante técnica de grupo nominal vía correo electrónico, 20 reactivos potenciales para la escala. Las respuestas se codificaron como datos dicotómicos "sí" o "no" según la claridad de la declaración.

Se eliminaron dos declaraciones después de dos rondas de revisión, por lo que el instrumento quedó integrado por 18 reactivos en formato de preguntas, para cuya respuestas se utilizó una escala tipo Likert (por ejemplo, "no", "no sé", "sí"). La escala se inició con una declaración general que define cuándo pensar en un origen orgánico o físico del síntoma.

\section{Paso 2, pilotaje}

La escala de 18 ítems se probó con 25 pacientes en consulta externa de diferentes especialidades para verificar la comprensión de los reactivos. No hubo cambios durante esta etapa.

\section{Paso 3, validación de la escala}

De abril de 2012 a mayo de 2013, se realizó un estudio de validación con una muestra de conveniencia de 400 pacientes. Se inscribieron e incluyeron en el estudio pacientes de 18 años o más, de uno y otro sexo, con diagnóstico de SFMNE (grupo $1=200$ ) 0 una enfermedad orgánica verificada médicamente (grupo 2 = 200). Los pacientes SFMNE fueron reclutados en una clínica de medicina familiar y en un servicio de psiquiatría de enlace del mismo hospital, donde fueron derivados de clínicas especializadas como traumatología y ortopedia, medicina interna, urología y otorrinolaringología, porque no se habían beneficiado de tratamientos anteriores y cumplían los criterios de Reid. ${ }^{6}$ El diagnóstico psiquiátrico previo o actual y haber recibido terapia familiar constituyeron los criterios de exclusión.

Los investigadores recopilaron datos demográficos y de características de los síntomas, previo consentimiento informado. La aplicación del instrumento fue realizada por uno de los investigadores y tuvo una duración aproximada de 10 minutos.
Se analizaron los datos en SPSS versión 23 para Windows. Se utilizó estadística descriptiva para determinar la frecuencias de las variables sociodemográficas y otras variables categóricas. Se utilizó la prueba t de Student en las variables continuas con distribución normal, con significación estadística de $p<0.05$.

La validez de constructo fue explorada mediante el análisis de componentes principales para el instrumento de 18,15 y 12 reactivos por separado, para determinar los valores de varianza explicada, consistencia interna y número de factores generados. Se usó el índice de Kaiser-Meyer-Olkin para el ajuste del análisis de componentes principales y la esfericidad de Bartlett. También se utilizó la rotación varimax (ortogonal) para optimizar la varianza.

Consideramos los siguientes criterios para la selección de factores:

- Autovalores superiores a 1 según la regla de Kaiser.

- Elementos con carga factorial superior a 0.40 para ser considerados significativos.

\section{Resultados}

No hubo datos perdidos como resultado de entrevistas directas. Las principales características sociodemográficas se muestran en la Tabla 1. Se encontraron diferencias significativas en el sexo (predominio del sexo femenino en el grupo SFMNE) y estado civil (mayor número de divorciados y en unión libre en el grupo no SFMNE), así como mayor proporción de desempleados en el grupo no SFMNE (Tabla 1).

En el grupo SFMNE predominaron los síntomas gastrointestinales, respiratorios y circulatorios con $26.5,12.5$ y $11.5 \%$ versus $18.0,3.5$ y $1.5 \%$ en el grupo con enfermedad orgánica $(p<0.001)$, en el cual la sintomatología general urinaria y del músculo esquelético fueron las más frecuentes $(p<0.001)$.

En el rango de respuestas, la respuesta "no sé" tuvo una frecuencia de 9 a $15 \%$; en la mayoría se trató de variables dicotómicas (sí/no).

\section{Análisis de componentes principales}

El valor observado en el índice de Kaiser-MeyerOlkin fue 0.899 , por lo tanto se consideró aconsejable realizar un análisis factorial de este conjunto de datos. El contraste de la esfericidad de Bartlett también fue altamente significativa $\left(\chi^{2}=1720.4, \mathrm{gl}=66, \mathrm{p}<0.001\right)$, lo que indica la presencia de correlaciones distintas de cero. 
Tabla 1. Características de la muestra

\begin{tabular}{|c|c|c|c|c|c|c|c|}
\hline \multirow[t]{2}{*}{ Variable } & \multicolumn{2}{|c|}{ SFMNE } & \multicolumn{2}{|c|}{ No SFMNE } & \multicolumn{2}{|c|}{ Total } & \multirow[t]{2}{*}{$p$} \\
\hline & $\%$ & $\mathrm{n}$ & $\%$ & $\mathrm{n}$ & $\%$ & $\mathrm{n}$ & \\
\hline \multirow{3}{*}{$\begin{array}{l}\text { Sexo } \\
\text { Hombre } \\
\text { Mujer }\end{array}$} & & & & & & & \multirow{3}{*}{0.041} \\
\hline & 52 & 26.0 & 69 & 34.0 & 121 & 30.3 & \\
\hline & 148 & 74.0 & 131 & 65.5 & 279 & 69.7 & \\
\hline \multicolumn{8}{|l|}{ Estado civil } \\
\hline Soltero & 54 & 27.0 & 57 & 28.5 & 111 & 27.8 & \multirow[t]{5}{*}{0.009} \\
\hline Casado & 82 & 41.0 & 108 & 54.0 & 190 & 47.5 & \\
\hline Viudo & 14 & 7.0 & 11 & 5.5 & 35 & 6.3 & \\
\hline Divorciado & 23 & 11.5 & 9 & 4.5 & 32 & 8.0 & \\
\hline Unión libre & 27 & 13.5 & 15 & 7.5 & 42 & 10.5 & \\
\hline \multicolumn{8}{|l|}{ Ocupación } \\
\hline Empleado & 67 & 33.5 & 83 & 41.5 & 150 & 37.5 & \multirow[t]{4}{*}{0.011} \\
\hline Ama de casa & 93 & 46.5 & 87 & 42.5 & 180 & 45.0 & \\
\hline Desempleado & 27 & 13.5 & 10 & 5.0 & 37 & 9.3 & \\
\hline Estudiante & 13 & 6.5 & 20 & 10.0 & 33 & 8.3 & \\
\hline \multirow{5}{*}{$\begin{array}{l}\text { Escolaridad } \\
\text { Primaria o } \\
\text { menor } \\
\text { Preparatoria } \\
\text { Licenciatura o } \\
\text { mayor }\end{array}$} & & & & & & & \multirow{4}{*}{0.90} \\
\hline & 61 & 30.5 & 62 & 31.0 & 123 & 30.8 & \\
\hline & 64 & 32 & 60 & 30.0 & 124 & 31.0 & \\
\hline & 75 & 37.5 & 78 & 39.0 & 153 & 38.3 & \\
\hline & Media & DE & Media & DE & & & \\
\hline $\begin{array}{l}\text { Edad } \\
\text { (años) }\end{array}$ & 41.2 & 16.1 & 42.4 & 15.6 & & & 0.466 \\
\hline
\end{tabular}

La varianza total explicada fue mayor en la escala de 12 reactivos (55\%); el análisis de componentes principales sugirió dos componentes con factores de carga superiores a 0.4 , con autovalores superiores a 1 (Tabla 2). En el factor 1 (atribución psicológica), de nueve reactivos, todas las cargas estuvieron entre 6 y 7; lo mismo ocurrió en el factor 2 (atribución orgánica), de tres reactivos. Se invirtió el valor de los tres reactivos orgánicos para realizar correlación entre reactivos y rotación, ya que se pretendía privilegiar la escala psicológica en lugar de la física.

La confiabilidad se analizó mediante alfa de Cronbach con base en ítems estandarizados. En general, el alfa de Cronbach fue de 0.853 para la escala de 12 ítems y para la escala psicológica (9) y orgánica (9) por separado fue de 0.886 y 0.682 , respectivamente, lo que corrobora una consistencia interna muy alta (Tabla 3). El factor 1 explicó 38.84 de la varianza y el factor 2, 16.21; ambos en total explicaron 55.06.

La matriz de correlación de las 12 variables muestra que 48 de las 66 correlaciones (72.7\%) fueron estadísticamente significativas $(p<0.01)$, lo que traduce que están altamente correlacionadas (Tabla 4).
Tabla 2. Estructura factorial con rotación varimax de la escala de atribución de síntomas médicamente no explicados

\begin{tabular}{l|c|c|}
\hline \multirow{2}{*}{ Variable } & \multicolumn{2}{|c|}{ Análisis factorial con 12 -reactivos } \\
\cline { 2 - 3 } & Factor 1 & Factor 2 \\
\hline X01Psi & 0.754 & -0.099 \\
\hline X02Psi & 0.756 & -0.061 \\
\hline X03Psi & 0.768 & -0.012 \\
\hline X05Psi & 0.678 & -0.095 \\
\hline X06Psi & 0.676 & -0.143 \\
\hline X07Psi & 0.673 & -0.205 \\
\hline X09Psi & 0.712 & -0.093 \\
\hline X10Psi & 0.751 & -0.093 \\
\hline X11Psi & 0.673 & -0.205 \\
\hline X04Org* & 0.298 & 0.679 \\
\hline X08Org* & 0.281 & 0.796 \\
\hline X12Org* & 0.301 & 0.720 \\
\hline Porcentaje & 40.665 & 55.068 \\
\hline $\begin{array}{l}\text { acumulado de } \\
\text { varianza explicada }\end{array}$ & & \\
\hline *Estos elementos fueron invertidos en su codificación. & \\
\hline Org: orgánico; Psy: psicológico. & & \\
\hline
\end{tabular}

En resumen, la escala Atribución del Síntomas en Pacientes con MUS (Medically Unexplained Symptoms) de 12 ítems parece ser fuerte y con buenas propiedades psicométricas, con el mayor porcentaje de la varianza total entre las diversas combinaciones de reactivos considerados.

\section{Discusión}

El predominio de SFMNE en el sexo femenino coincidió con lo reportado en la literatura. ${ }^{19}$ En el grupo SFMNE predominaron los síntomas gastrointestinales, respiratorios y circulatorios, a diferencia de lo reportado por Lee en 2016, quien encontró predominio de problemas neurológicos o respiratorios. ${ }^{7}$

La mayor proporción de desempleados en el grupo SFMNE se explica por los problemas económicos derivados de la atención médica a largo plazo de estos pacientes, ${ }^{20}$ además del efecto negativo por las ausencias laborales debido a las enfermedades de larga duración. ${ }^{21}$

Se ha reiterado la importancia de desarrollar intervenciones terapéuticas factibles, sencillas y efectivas para SFMNE. El modelo de atribución para identificar 


\begin{tabular}{|c|c|c|c|c|c|c|c|c|}
\hline \multirow[t]{2}{*}{ Componente } & \multicolumn{2}{|c|}{ Autovalores iniciales } & \multicolumn{3}{|c|}{$\begin{array}{l}\text { Suma de las saturaciones de la } \\
\text { extracción al cuadrado }\end{array}$} & \multicolumn{3}{|c|}{$\begin{array}{c}\text { Suma de saturaciones de la rotación al } \\
\text { cuadrado }\end{array}$} \\
\hline & Total & $\%$ de varianza & $\%$ acumulado & Total & $\%$ de varianza & Total & $\%$ de varianza & $\%$ acumulado \\
\hline 1 & 4.880 & 40.665 & 40.665 & 4.880 & 40.665 & 4.662 & 38.849 & 38.849 \\
\hline 2 & 1.728 & 14.403 & 55.068 & 1.728 & 14.403 & 1.946 & 16.219 & 55.068 \\
\hline 3 & 0.820 & 6.835 & 61.903 & & & & & \\
\hline 4 & 0.669 & 5.576 & 67.479 & & & & & \\
\hline 5 & 0.631 & 5.259 & 72.738 & & & & & \\
\hline 6 & 0.607 & 5.056 & 77.794 & & & & & \\
\hline 7 & 0.545 & 4.540 & 82.334 & & & & & \\
\hline 8 & 0.519 & 4.329 & 86.662 & & & & & \\
\hline 9 & 0.431 & 3.592 & 90.254 & & & & & \\
\hline 10 & 0.418 & 3.484 & 93.738 & & & & & \\
\hline 11 & 0.396 & 3.303 & 97.041 & & & & & \\
\hline 12 & 0.355 & 2.959 & 100.000 & & & & & \\
\hline
\end{tabular}

Tabla 4. Matriz de correlación de Pearson de una muestra de 400 participantes. Escala de 12 reactivos

\begin{tabular}{|c|c|c|c|c|c|c|c|c|c|c|c|c|}
\hline & $\begin{array}{l}\text { X01 } \\
\text { Psi }\end{array}$ & $\begin{array}{l}\text { X02 } \\
\text { Psi }\end{array}$ & $\begin{array}{l}\text { X03 } \\
\text { Psi }\end{array}$ & $\begin{array}{l}\text { X05 } \\
\text { Psi }\end{array}$ & $\begin{array}{l}\text { X06 } \\
\text { Psi }\end{array}$ & $\begin{array}{l}\text { X07 } \\
\text { Psi }\end{array}$ & $\begin{array}{l}\text { X09 } \\
\text { Psi }\end{array}$ & $\begin{array}{l}\text { X10 } \\
\text { Psi }\end{array}$ & $\begin{array}{l}X 11 \\
\text { Psi }\end{array}$ & $\begin{array}{l}\text { X04 } \\
\text { Org }\end{array}$ & $\begin{array}{l}\text { X08 } \\
\text { Org }\end{array}$ & $\begin{array}{l}\text { X12 } \\
\text { Org }\end{array}$ \\
\hline X01Psi & 0.9286 & & & & & & & & & & & \\
\hline X02Psi & 0.001 & 0.8739 & & & & & & & & & & \\
\hline X03Psi & 0.001 & 0.001 & 0.8354 & & & & & & & & & \\
\hline X05Psi & 0.001 & 0.001 & 0.001 & 0.8106 & & & & & & & & \\
\hline X06Psi & 0.001 & 0.001 & 0.001 & 0.001 & 0.7979 & & & & & & & \\
\hline X07Psi & 0.001 & 0.001 & 0.001 & 0.001 & 0.001 & 0.8161 & & & & & & \\
\hline X09Psi & 0.001 & 0.001 & 0.001 & 0.001 & 0.001 & 0.001 & 0.8675 & & & & & \\
\hline X10Psi & 0.001 & 0.001 & 0.001 & 0.001 & 0.001 & 0.001 & 0.001 & 0.9195 & & & & \\
\hline X11Psi & 0.001 & 0.001 & 0.001 & 0.001 & 0.001 & 0.001 & 0.001 & 0.001 & 0.9069 & & & \\
\hline X04Org* & 0.001 & 0.001 & 0.001 & 0.010 & 0.048 & 0.053 & 0.005 & 0.002 & 0.023 & 0.8519 & & \\
\hline X080rg* & 0.044 & 0.001 & 0.001 & 0.015 & 0.022 & 0.459 & 0.011 & 0.029 & 0.015 & 0.001 & 0.8941 & \\
\hline X12Org* & 0.001 & 0.005 & 0.001 & 0.012 & 0.021 & 0.045 & 0.003 & 0.001 & 0.031 & 0.001 & 0.001 & 0.8871 \\
\hline Media & 1.783 & 1.615 & 1.563 & 1.568 & 1.505 & 1.575 & 1.620 & 1.768 & 1.723 & 1.605 & 1.740 & 1.700 \\
\hline
\end{tabular}

*Estos elementos fueron invertidos en su codificación.

Org: orgánico; Psy: psicológico.

si el origen del síntoma es psicológico tiene resultados mixtos e, incluso, se ha considerado innecesario; sin embargo, falta una estrategia metodológica para aplicarlo. ${ }^{22}$ Se ha demostrado su utilidad cuando lo emplean profesionales sin experiencia psiquiátrica en medicina de atención primaria. ${ }^{23}$

Por ello, los médicos de familia necesitan una herramienta para facilitar el tratamiento de estos pacientes 
con SFMNE. La relevancia radica en ayudarlos a comprender el origen de sus síntomas. La literatura revela que cuando a los pacientes se les pregunta directamente sobre la causa, la mayoría opta por atribuciones "normalizadoras", que tienden a minimizar la importancia de los síntomas y vincularlos con causas ambientales o contextuales como comer en exceso o hacer ejercicio en exceso, entre otras. ${ }^{17}$ Esta respuesta neutral podría corresponder a lo que obtuvimos en nuestro estudio como la respuesta "no sé"; sin embargo, el porcentaje más alto fue una respuesta dicotómica de sí/no a la atribución psicosocial.

Esta es la primera escala reportada en la literatura que mide la atribución del paciente de síntomas inespecíficos; constituye una herramienta rápida y estructurada que podría ayudar a establecer un vínculo entre incidentes y experiencias personales, emociones o estresores psicosociales de los pacientes y sus dolencias físicas, facilitar el proceso de aceptación y conducir a una mejor atención clínica del paciente.

El instrumento se puede utilizar en cualquier etapa de la interacción con el paciente y podría brindar una oportunidad para la expresión de los síntomas. Además, ayuda al médico a crear un vínculo entre el evento estresante y el síntoma inespecífico, siempre que se aborde la mayoría de los elementos del componente psicosocial.

Recientemente otro autor demostró la estabilidad temporal de la escala (test-retest) con un valor alto de coeficiente de correlación intraclase. ${ }^{24}$ Sin embargo, es necesario consolidar la validez del constructo utilizando técnicas de análisis factorial confirmatorio que permitan estimar simultáneamente la contribución de cada ítem a una o más dimensiones, así como la estimación de posibles correlaciones entre las dimensiones.

Se reconocen algunas limitaciones de este estudio: la falta de uniformidad en el diagnóstico de los pacientes con SFMNE es una limitación en sí misma, ya que ningún procedimiento para identificar SFMNE ha sido ampliamente aceptado. Para este estudio utilizamos los criterios publicados por Reid et al., y los de Smith y Dwamena para clasificar sistemática y consistentemente a todos los participantes. , $^{6}$

\section{Conclusiones}

La escala ASPAMUS parece prometedora como herramienta para facilitar el manejo psicosocial de los pacientes con SFMNE. Su validez de constructo ha sido probada y la consistencia interna es adecuada; en investigaciones posteriores, también se demostró la estabilidad temporal.

Se necesitan más estudios para validar a fondo la escala mediante análisis factorial confirmatorio y verificar su utilidad clínica en la identificación de pacientes con SFMNE que pueden beneficiarse de las intervenciones conductuales. Sin embargo, hay mucho campo por explorar sobre el mejor abordaje para tratar a los pacientes con SFMNE y abre líneas de investigación para desarrollar en el futuro.

\section{Agradecimientos}

Los autores agradecen al personal académico y administrativo, así como a los residentes del Hospital Universitario "Dr. José Eleuterio González" y al doctor Sergio Lozano Rodríguez, por su ayuda en la traducción y edición del manuscrito.

\section{Financiamiento}

La presente investigación no recibió ninguna beca específica de agencias de los sectores públicos, comercial, o sin ánimo de lucro.

\section{Conflicto de intereses}

Los autores declaran no tener conflicto de intereses.

\section{Responsabilidades éticas}

Protección de personas y animales. Los autores declaran que para esta investigación no se realizaron experimentos en seres humanos ni en animales.

Confidencialidad de los datos. Los autores declaran que siguieron los protocolos de su centro de trabajo sobre la publicación de datos de pacientes.

Derecho a la privacidad y consentimiento informado. Los autores declaran que en este artículo no aparecen datos de pacientes.

\section{Bibliografía}

1. López-Santiago J, Belloch A. El laberinto de la somatización: se buscan salidas. Rev Psicopatol Psicol Clin. 2012;17:151-172.

2. Granel A. Pacientes con síntomas somáticos no explicables. Evid Actual Pract Ambul. 2006;9:176-180.

3. Salazar de la Cruz M, Jurado-Vega A. Niveles de intervención familiar. Acta Med Peruana. 2013;30:37-41.

4. Husain $M$, Chalder T. Medically unexplained symptoms: assessment and management. Clinical Med (Lond). 2021;2:13-18.

5. Haller H, Cramer H, Lauche R, Dobos G. Somatoform disorders and medically unexplained symptoms in primary care. Dtsch Arztebl Int. 2015;112:279-287 
6. Reid S, Wessely S, Crayford T, Hotopf M. Frequent attenders with medically unexplained symptoms: service use and costs in secondary care. Br J Psychiatry. 2002;180:248-253.

7. Lee K, Johnson MH, Harris J, Sundram F. The resource utilization of medically unexplained physical symptoms. SAGE Open Med. 2016;4:2050312116666217.

8. Ramírez-Aranda JM, Loera-Manzanares SL, Garza-Elizondo T, Gómez-Gómez C. Percepción del estrés en pacientes con síntomas físicos médicamente inexplicados. Aten Fam. 2013;20:118-122.

9. De la Rosa-Melchor D, Ramírez-Aranda JM, Garza-Elizondo T, Riquelme-Heras HM, Rodríguez-Rodríguez I, Durán-Gutiérrez RA. Eficacia de la terapia de resolución de problemas en pacientes con síntomas físicos médicamente no explicados. Aten Fam. 2013;20:12-15.

10. Rangel BE. Eficacia de una intervención psicosocial en el abordaje de pacientes con SMI (síntomas físicos medicamente inexplicados) [Tesis]. México: Universidad Autónoma de Nuevo León; 2011.

11. Garza-Elizondo T, Gutiérrez-Herrera R. Trabajo con familias. Abordaje médico integral. México: El Manual Moderno; 2015.

12. Menon V, Rajan TM, Kuppili PP, Sarkar S. Cognitive behavior therapy for medically unexplained symptoms: A systematic review and meta-analysis of published controlled trials. Indian J Psychol Med. 2017;39:399-406.

13. Liu J, Gill NS, Teodorczuk A, Li ZJ, Sun J. The efficacy of cognitive behavioural therapy in somatoform disorders and medically unexplained physical symptoms: A meta-analysis of randomized controlled trials. J Affect Disord. 2019;245:98-112.

14. Robbins JM, Kirmayer LJ. Attributions of common somatic symptoms. Psychol Med. 1991;21:1029-1045.

15. Aronson KR. The reliability and validity of the symptom interpretation questionnaire. J Psychosom Res. 2006;61:807-811.
16. Kurlansik SL, Maffei MS. Somatic symptom disorder. Am Fam Physician. 2016:93:49-54.

17. Kessler D, Heath I, Lloyd K, Lewis G, Gray DP. Cross sectional study of symptom attribution and recognition of depression and anxiety in primary care. BMJ. 1999;318:436-440.

18. Smith RC, Dwamena FC. Classification and diagnosis of patients with medically unexplained symptoms. J Gen Intern Med. 2007;22:685-691.

19. Andersen NL, Eplov LF, Andersen JT, Hjorthoj CR, Birket-Smith M. Health care use by patients with somatoform disorders: a register-based follow-up study. Psychosomatics. 2013;54:132-141.

20. Konnopka A, Kaufmann C, König HH, Heider D, Wild B, Szecsenyi J, et al. Association of costs with somatic symptom severity in patients with medically unexplained symptoms. J Psychosom Res. 2013;75:370-375.

21. Loengaard K, Bjorner JB, Fink PK, Burr H, Rugulies R. Medically unexplained symptoms and the risk of loss of labor market participation--a prospective study in the Danish population. BMC Public Health. 2015;15:844.

22. Gask L, Dowrick C, Salmon P, Peters S, Morriss R. Reattribution reconsidered: narrative review and reflections on an educational intervention for medically unexplained symptoms in primary care settings. J Psychosom Res. 2011;71:325-334.

23. Tschudi-Madsen $H$, Kjeldsberg M, Natvig B, et al. Multiple symptoms and medically unexplained symptoms--closely related concepts in general practitioners' evaluations. A linked doctor-patient study. J Psychosom Res. 2013;74:186-190.

24. Frías-Gómez VL. Validación y test-retest de la escala para percepción del origen del síntoma en pacientes con síntomas físicos medicamente inexplicables [Tesis]. México: Universidad Autónoma de Nuevo León; 2018. 